\title{
Microbial Biomarkers in Patients with Nonresponsive Celiac Disease
}

\section{Viitasalo, Liisa}

2018-12

Viitasalo , L , Kurppa , K , Ashorn , M , Saavalainen , P , Huhtala , H, Ashorn , S , Mäki , M , Ilus , T , Kaukinen , K \& Iltanen , S 2018 , ' Microbial Biomarkers in Patients with Nonresponsive Celiac Disease ' , Digestive Diseases and Sciences , vol. 63 , no. 12 , pp. 3434-3441 . https://doi.org/10.1007/s10620-018-5285-z

http://hdl.handle.net/10138/276968

https://doi.org/10.1007/s10620-018-5285-z

publishedVersion

Downloaded from Helda, University of Helsinki institutional repository.

This is an electronic reprint of the original article.

This reprint may differ from the original in pagination and typographic detail.

Please cite the original version. 


\title{
Microbial Biomarkers in Patients with Nonresponsive Celiac Disease
}

\author{
Liisa Viitasalo ${ }^{1,2} \cdot$ Kalle Kurppa $^{1}$ (1) $\cdot$ Merja Ashorn ${ }^{1} \cdot$ Päivi Saavalainen $^{3} \cdot$ Heini Huhtala $^{4} \cdot$ Sara Ashorn ${ }^{1}$. \\ Markku Mäki $^{1} \cdot$ Tuire Ilus $^{5} \cdot$ Katri Kaukinen $^{6,7} \cdot$ Sari Iltanen $^{1,8}$
}

Received: 3 April 2018 / Accepted: 11 September 2018 / Published online: 20 September 2018

(c) Springer Science+Business Media, LLC, part of Springer Nature 2018

\begin{abstract}
Background and Aims In nonresponsive celiac disease (NRCD), the symptoms and duodenal damage persist despite a gluten-free diet. Celiac disease patients with persistent symptoms are found to have a dysbiotic microbiota. We thus hypothesized that increased seroreactivity to the serum gluten-sensitive microbial antibodies Saccharomyces cerevisiae (ASCA), Pseudomonas fluorescens-associated sequence (I2), and Bacteroides caccae TonB-linked outer membrane protein (OmpW) is associated with NRCD.

Methods ASCA, I2 and OmpW were measured in 20 seronegative CD patients with persistent villous damage despite strict dietary treatment (NRCD group). Fifty-eight responsive patients served as CD controls (55 on gluten-free treatment) and 80 blood donors as non-CD controls.

Results At least one microbial marker was positive in $80 \%$ of NRCD patients, in $97 \%$ of untreated CD and $87 \%$ of treated CD patients, and in $44 \%$ of controls. NRCD patients had the highest frequency of ASCA positivity $(65 \%$ vs 52,20 , and $0 \%$, respectively) and also significantly higher ASCA IgA (median $14.5 \mathrm{U} / \mathrm{ml})$ and IgG $(32.5 \mathrm{U} / \mathrm{ml})$ titers than treated CD patients $(7.0 \mathrm{U} / \mathrm{ml}, 13.0 \mathrm{U} / \mathrm{ml})$ and non-CD controls $(4.5 \mathrm{U} / \mathrm{ml}, 5.8 \mathrm{U} / \mathrm{ml})$. The frequencies of $\mathrm{I} 2$ and OmpW were lower in NRCD than in untreated CD (65\% and $45 \%$ vs $86 \%$ and $59 \%$, respectively), and I2 titers were higher in NRCD (median absorbance 0.76) and untreated (1.0) and treated (0.83) CD than controls (0.32). OmpW was elevated in untreated (1.1) and treated (0.94) CD patients compared with controls (0.79).

Conclusions Seropositivity and high titers of ASCA are associated with NRCD and might serve as an additional follow-up tool in CD.
\end{abstract}

Keywords Nonresponsive celiac disease $\cdot$ Microbiota $\cdot$ Saccharomyces cerevisiae $\cdot$ Pseudomonas fluorescence $\cdot$

Bacteroides caccae

\section{Introduction}

Celiac disease (CD) is an immune-mediated intestinal disease characterized by diverse symptoms, specific autoantibodies against tissue transglutaminase (tTG-ab), and smallbowel mucosal injury. The only cure is a strict gluten-free diet (GFD), initiation of which usually results in beneficial response. Some patients, however, have nonresponsive celiac disease (NRCD) characterized by persistent clinical symptoms and histological damage [1-3]. The most common reason for NRCD is ongoing gluten intake, but there may also be coexisting disorders such as irritable bowel syndrome

Kalle Kurppa

kalle.kurppa@uta.fi

Extended author information available on the last page of the article and microscopic colitis sustaining the symptoms [1-3]. After exclusion of other etiologies, refractory celiac disease (RCD) must be ruled out. Suggested risk factors for RCD are for example older age, male gender, and seronegativity at diagnosis [4], but the fundamental reasons for the development of RCD remain unknown.

Recently, there has been growing interest in the role of abnormal gut microbiota in the development of chronic intestinal diseases [5, 6]. Alongside the well-known association with anti-Saccharomyces cerevisiae antibodies (ASCA), we and others have reported increased serological responses to Pseudomonas fluorescens-associated sequence (I2) and Bacteroides caccae TonB-linked outer membrane protein $(\mathrm{OmpW})$ in IBD [7-9]. A dysbiotic microbiota has also been associated with persistent symptoms in treated CD [10] and presence of ASCA and I2 with an increased risk of a complicated course 
in IBD [11-14]. These microbial markers are also gluten-sensitive and already present in early stages of $\mathrm{CD}$, even before the development of villous atrophy and serum CD-specific autoantibodies (tTG-ab) [15-18].

Based on these observations, we hypothesized that NRCD could be associated with a dysbiotic intestinal microbiota reflected by increased seroreactivity against ASCA, I2, and OmpW. In addition, since most RCD patients are negative for CD autoantibodies [19], microbial markers might serve as novel noninvasive diagnostic and follow-up tools. We tested these hypotheses by comparing microbial seroreactivity between groups of NRCD patients, responsive CD patients, and non-CD controls.

\section{Methods}

\section{Patients and Study Design}

The study was conducted in Tampere University and Tampere University Hospital. The cohort comprised 20 adult NRCD patients, $58 \mathrm{CD}$ patients with dietary response to GFD, and 80 healthy blood donors (non-CD controls). CD patients underwent careful clinical, serological, and histological evaluation during the diagnostic visit, and additional blood samples were drawn for serological analyses and HLA genotyping. After the diagnosis, the patients started a GFD under the supervision of a professional dietitian, and upper gastrointestinal endoscopy and clinical assessment were carried out after 1 year. The NRCD group comprised $20 \mathrm{CD}$ patients who continued to have persistent clinical symptoms and villous atrophy despite long-term (median 3.5 years) strict GFD. Besides the esogastroduodenoscopy, the patients underwent various other medical investigations such as laboratory testing, abdominal imaging, and colonoscopy to exclude other than dietary reasons for NRCD [20]. Repeat endoscopy was conducted after 1 year in 15 NRCD patients, whereas the rest five were biopsied within $2-4$ years. Two of the NRCD patients developed a true refractory CD with abnormal intraepithelial lymphocyte phenotype during the follow-up. The $58 \mathrm{CD}$ patients who evinced a clear clinical, serological, and histological response to GFD represented the responsive $\mathrm{CD}$ patients. Among these, control sera for microbial analyses after 1 year on GFD were available from 55 subjects.

The Ethical Committee of the Pirkanmaa Hospital District approved the study protocol and patient enrollment. All participants gave written informed consent.

\section{Small-Bowel Mucosal Morphology and Immunohistochemistry}

Upper gastrointestinal endoscopy with multiple duodenal biopsies was undertaken in CD and NRCD patients at diagnosis and on GFD. The biopsy samples were orientated and processed according to our standard operating procedures [21]. Mucosal inflammation and villous height-crypt depth ratio $(\mathrm{VH} / \mathrm{CrD})$ were calculated from $\mathrm{H} \& \mathrm{E}$-stained paraffin sections as an average from at least three separate villous-crypt units. In addition, separate biopsies were taken for immunohistochemical analyses, embedded in optimal cutting temperature compound (Miles Labs, Elkhart, IN, USA) and stored at $-80{ }^{\circ} \mathrm{C}$ until further used. Cryostat sections were cut $5 \mu \mathrm{m}$ thick and processed for immunohistochemistry as described elsewhere [22]. The densities of mucosal $\mathrm{CD} 3+$ and $\gamma \delta+$ IELs were quantified from the frozen sections utilizing monoclonal antibodies against CD3 (Leu-4; Becton Dickinson, San Jose, CA) and TCR $\gamma$ (Endogen, Woburn, MA) [22]. All evaluations were carried out without previous information on the patient's medical history or laboratory results.

\section{Serum Antibody Tests and HLA-Typing}

Serum for the celiac autoantibodies and microbial markers were drawn in connection with the endoscopy visits. Serum IgA class tissue transglutaminase antibodies (tTG-ab) were measured by a commercial enzyme-linked immunosorbent assay (ELISA) according to the manufacturer's instructions (Celikey; Phadia, Freiburg, Germany). Serum tTG-ab values $\geq 5.0 \mathrm{U} / \mathrm{ml}$ were considered positive. In case of selective IgA deficiency, the serum tests were carried out utilizing the corresponding $\mathrm{IgG}$ class antibodies.

Serum IgA and IgG class ASCA were measured by ELISA (Quanta Lite ASCA; INOVA Diagnostics Inc., San Diego, CA) according to manufacturer's protocol. Results $\geq 25 \mathrm{U} / \mathrm{ml}$ for IgA and IgG ASCA were regarded as positive [15]. I2 and OmpW were expressed in E. coli XL-1 blue and E. coli BL-21 (Stratagene, La Jolla, CA) strains, and antigens for the corresponding IgA ELISA test were produced using previously reported antigen purification techniques [23, 24]. The cutoff level for positivity was set at absorbance 0.5 for I2 and at 1.0 for OmpW [7].

The presence of the celiac disease-associated HLA alleles (HLA)-DQB $1 * 02$ and DQB $1 * 0302$ (DQ2 and DQ8) was analyzed in whole blood samples from all NRCD and CD patients as described elsewhere [25].

\section{Statistical Analysis}

Quantitative data were expressed as percentages or as medians with lower and upper quartiles. Kruskal-Wallis and Mann-Whitney tests were used to compare serum antibody titers and other parameters between the groups, and Dunn-Bonferroni test was used for post hoc pairwise comparisons. When comparing seropositivity to ASCA, I2, and $\mathrm{OmpW}$, the groups were adjusted by age and gender by 
binary logistic regression. To express overlapping of seropositivity for different microbial antibodies, the data were cross-tabulated. A $p$ value $<0.05$ was considered significant. Statistical analyses were carried out with IBM SPSS Statistics, version 23.

\section{Results}

Patients with NRCD were somewhat older and non-CD controls more often men than subjects in the other study groups (Table 1). However, adjusting by age and gender did not change the study results. Coexisting collagenous colitis was diagnosed in two and unspecific proctitis in one NRCD patients, but treatment of these diseases did not affect the $\mathrm{CD}$ symptoms or duodenal histology. The exact tTG-ab value at diagnosis was available from nine NRCD patients of whom in eight it was positive. Their median value was somewhat higher compared with GFD-responsive patients, but the difference was not significant (72.6 vs $31.7 \mathrm{U} / 1$, respectively, $p=0.125$ ). Furthermore, $\mathrm{VH} / \mathrm{CrD}$ was lower in NRCD than GFD-responsive patients at diagnosis with borderline significance (median ratio 0.2 vs 0.4 , respectively, $p=0.065$ ).

By definition, on a GFD NRCD patients had lower VH/ CrD than CD patients (Table 1). Morphology of the duodenal mucosa was fully normalized in 32 (58\%) out of the 55 GFD-responsive patients after 1 year on GFD, while the rest had ongoing recovery. NRCD patients had the lowest median tTG-ab value of all groups (the value was immeasurably low in 16 out of 20 cases), but they still had median CD3 + and $\gamma \delta+$ IEL densities almost as high as untreated CD patients (Table 1). The tTG-ab titers clearly decreased on GFD but remained marginally elevated in seven GFDresponsive patients, as well as in four NRCD patients. One of the NRCD patients had IgA deficiency and also negative IgG class tTG-ab on GFD. All of the controls had negative tTG-ab. All NRCD and CD patients had the celiac diseaseassociated HLA-DQ2 and/or DQ8.
Seropositivity for at least one of the three microbial markers (ASCA, I2, OmpW) was seen in $80 \%$ of NRCD patients, $97 \%$ of untreated and $87 \%$ of treated CD patients, and in $44 \%$ of non-CD controls (Fig. 1). The most conspicuous difference between the groups was observed in ASCA, seropositivity for which was even more common in NRCD (65\%) than untreated CD (52\%), while only $20 \%$ of the treated CD patients and none of the controls were ASCA positive (Fig. 1). Seropositivity to I2 and OmpW was significantly more common in untreated CD patients ( $86 \%$ and $59 \%$, respectively) and less frequent in controls ( $31 \%$ and 24\%) (Fig. 1), whereas no significant difference was seen between NRCD and other groups.

NRCD patients had significantly higher IgA and IgG class ASCA titers compared with responsive CD patients on a GFD and non-CD controls; the titers were also higher in untreated CD patients than in those on a GFD (Figs. 2a and 2b). Although the difference was not clinically significant, the ASCA titer medians were higher in NRCD (ASCA IgA 14.5 and $\operatorname{IgG} 32.5)$ than in untreated CD (10.5 and 23.5, respectively). I 2 titers were significantly higher in NRCD and both $\mathrm{CD}$ groups compared with non-CD controls, whereas there was no significant difference between the NRCD and CD groups (Fig. 2c). OmpW titers did not differ significantly between NRCD and CD groups, but were higher in both untreated and treated CD compared with nonCD controls (Fig. 2d).

There was no correlation between age and any of the serum antibody titers within the four subgroups. In addition, the titers did not differ significantly when genders were evaluated separately (data not shown).

\section{Discussion}

The most conspicuous finding in the present study was the highest frequency of ASCA positivity in NRCD patients compared with the other study groups. In accord, NRCD

Table 1 Demographic, histological and serological data in nonresponsive and responsive celiac disease (CD) patients and in nonceliac controls

\begin{tabular}{|c|c|c|c|c|}
\hline & $\begin{array}{l}\text { Nonresponsive CD } \\
n=20\end{array}$ & $\begin{array}{l}\mathrm{CD} \text { at diagnosis } \\
n=58\end{array}$ & $\begin{array}{l}\text { CD on a GFD } \\
n=55\end{array}$ & $\begin{array}{l}\text { Controls } \\
n=80\end{array}$ \\
\hline Females, $\%$ & 85.0 & 77.6 & 76.4 & 35.0 \\
\hline Age, median $\left(\mathrm{Q}_{1-3}\right)$, years & $50(43-67)$ & $45(36-59)$ & $46(38-60)$ & $41(31-56)$ \\
\hline $\mathrm{VH} / \mathrm{CrD}$, median $\left(\mathrm{Q}_{1-3}\right)$ & $0.3(0.1-1.0)$ & $0.4(0.2-0.7)$ & $2.1(1.5-2.6)^{\mathrm{a}}$ & ND \\
\hline $\mathrm{CD} 3$ + IELs, median $\left(\mathrm{Q}_{1-3}\right)$, cells $/ \mathrm{mm}$ & $38.0(26.0-55.0)$ & $51.0(35.5-69.0)$ & $28.0(19.5-37.5)$ & ND \\
\hline$\gamma \delta+$ IELs, median $\left(\mathrm{Q}_{1-3}\right)$, cells $/ \mathrm{mm}$ & $11.9(4.7-16.8)$ & $13.4(9.0-17.2)$ & $8.7(4.7-12.7)$ & ND \\
\hline $\mathrm{TG} 2 \mathrm{ab}$, median $\left(\mathrm{Q}_{1-3}\right), \mathrm{U} / \mathrm{l}$ & $0.0(0.0-0.0)$ & $31.7(8.7-71.6)$ & $1.9(1.1-3.6)$ & $0.3(0.1-0.5)$ \\
\hline
\end{tabular}

$G F D$ gluten-free diet, $V H / C r D$ villous height-crypt depth ratio, $Q_{l-3}$ lower and upper quartiles, $T G 2 a b$ transglutaminase 2 antibodies, $I E L$ intraepithelial lymphocyte, $N D$ no data

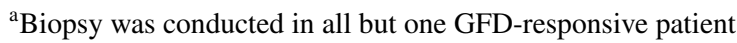




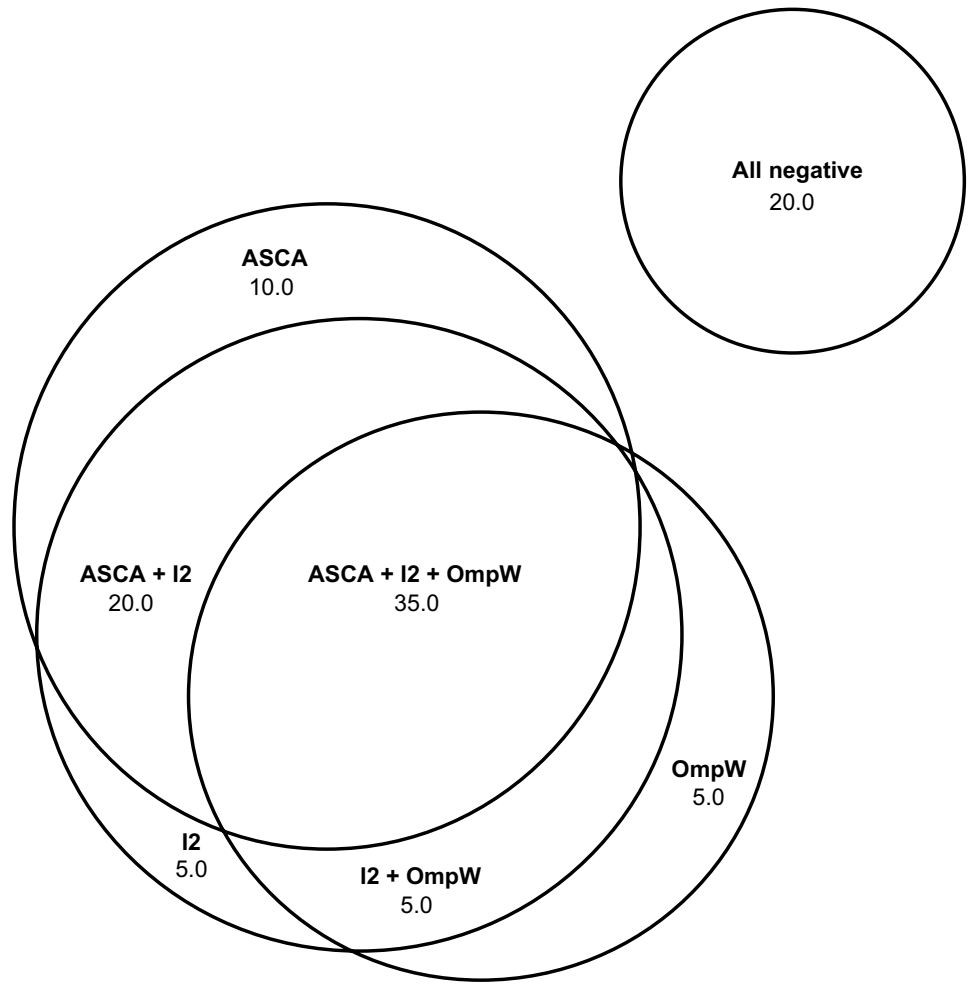

Nonresponsive CD ( $n=20)$

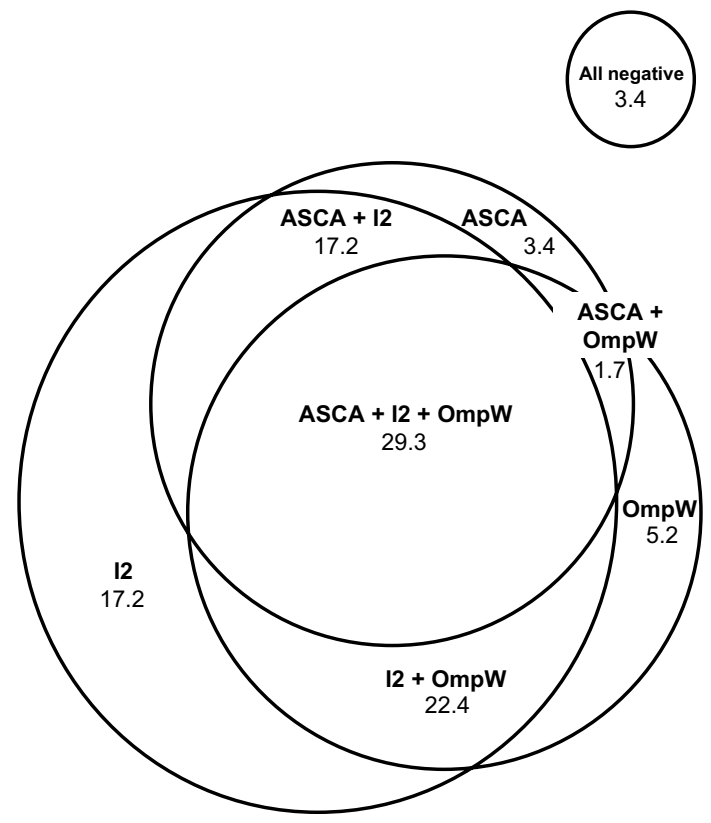

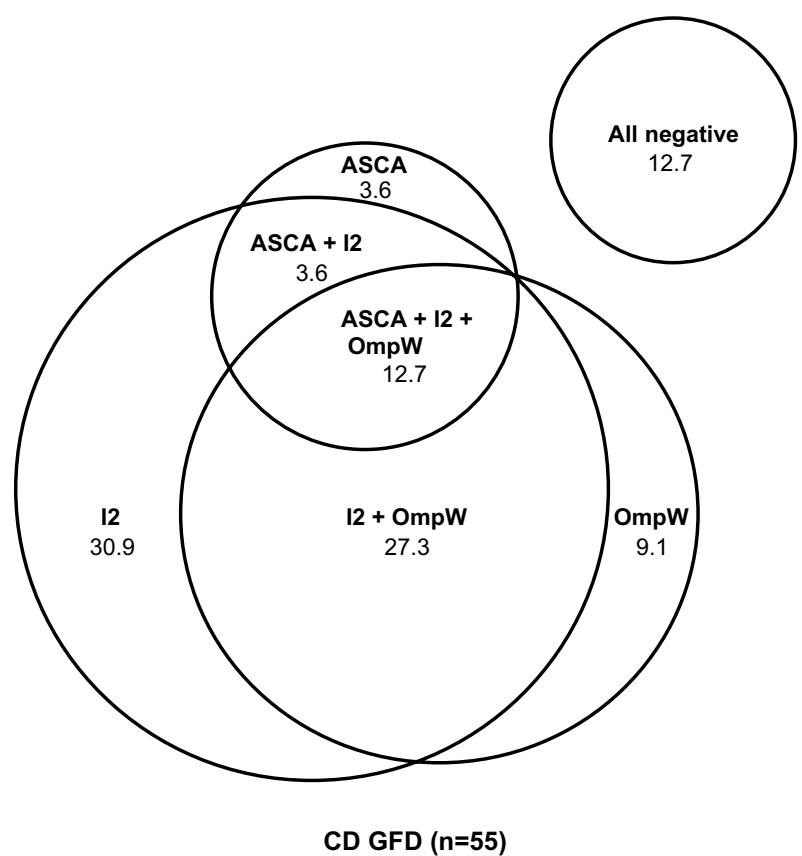

Fig. 1 Distribution of positivity to Saccharomyces cerevisiae (ASCA), Pseudomonas fluorescens-associated sequence (I2) and Bacteroides caccae TonB-linked outer membrane protein $(\mathrm{OmpW})$ serum
CD diagnosis $(n=58)$
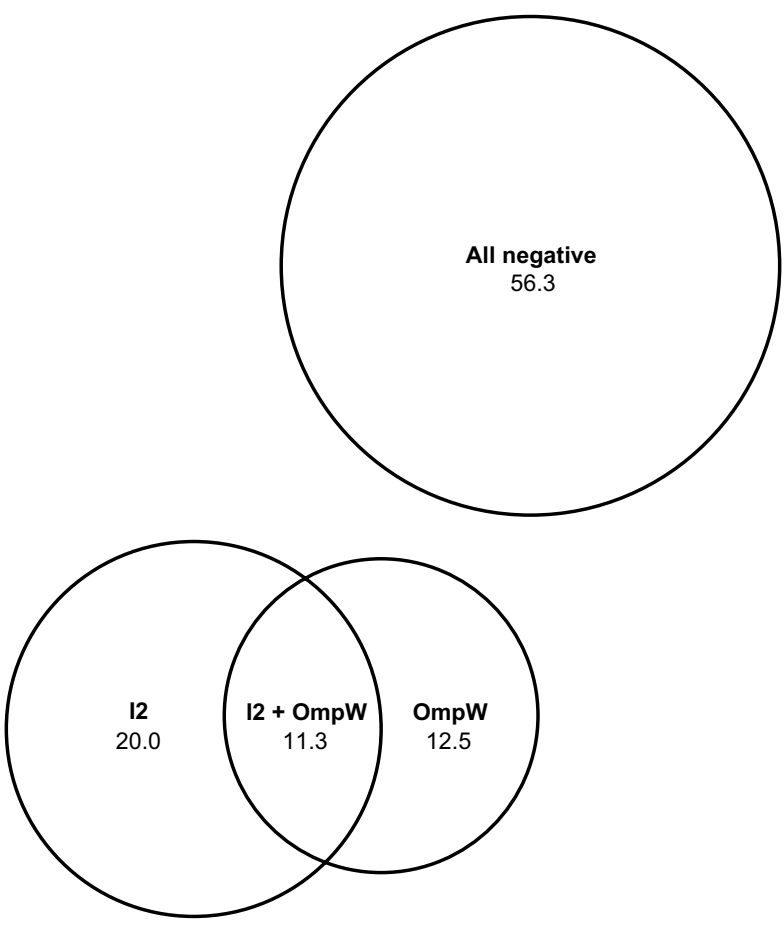

Control $(n=80)$

antibodies in NRCD and CD (at the time of diagnosis and on a GFD) patients and controls. The numbers are given as percentages for each antibody and their combinations (together the numbers add 100\%) 


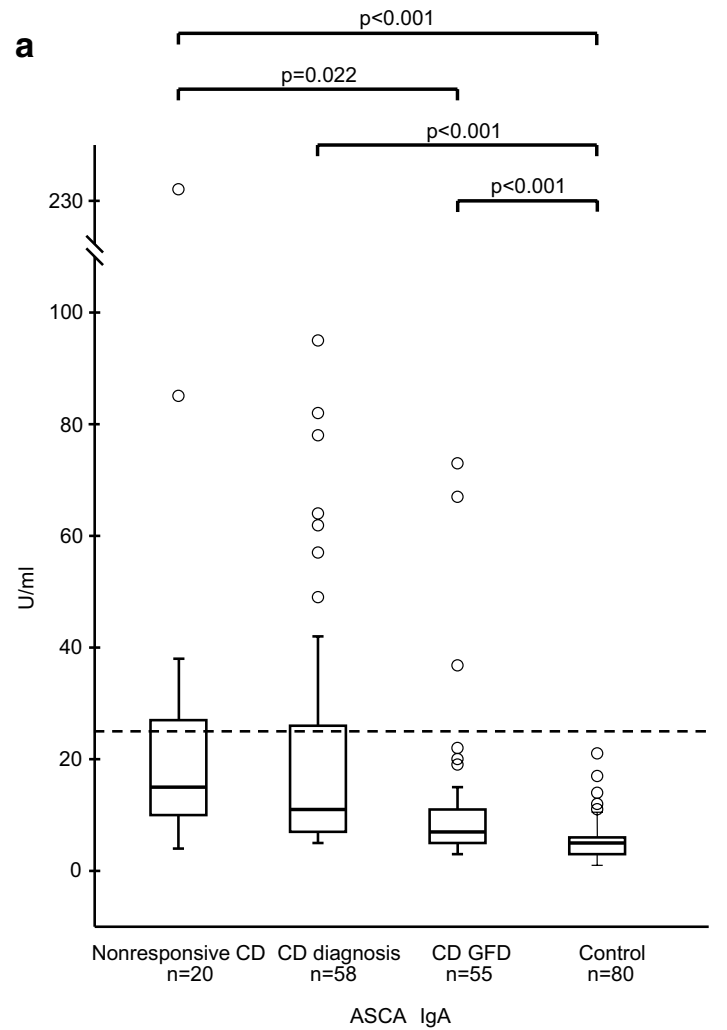

C
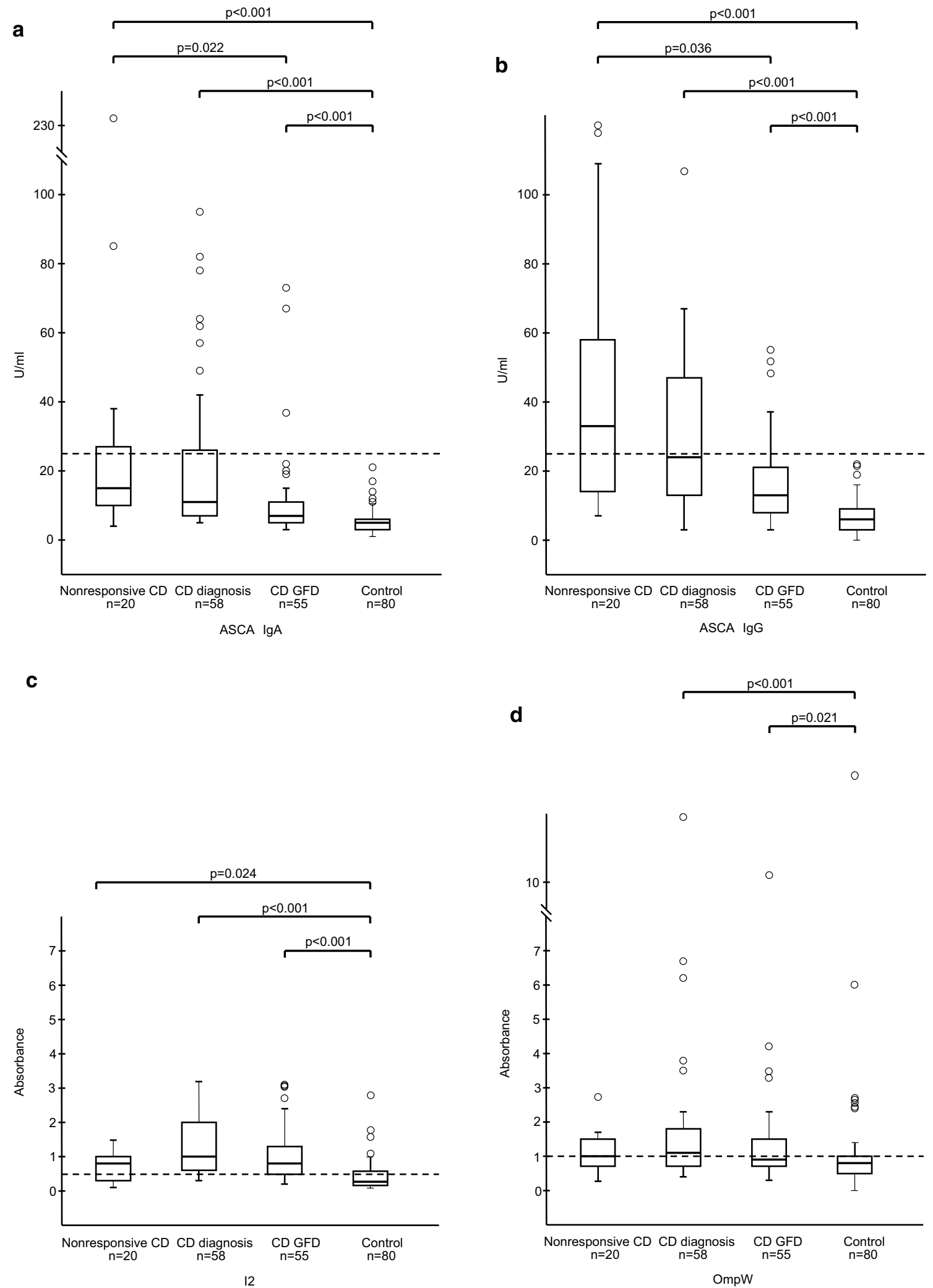

Fig. 2 Serum titers of Saccharomyces cerevisiae antibodies (ASCA) in $\operatorname{IgA}$ (a) and $\operatorname{IgG}$ (b) classes, Pseudomonas fluorescens-associated sequence (I2) (c) and Bacteroides caccae TonB-linked outer mem-

brane protein $(\mathrm{OmpW})(\mathbf{d})$ antibodies. Horizontal lines indicate the cutoff level for seropositivity of each antibody 
patients also showed higher median ASCA levels than the GFD-responsive CD patients and non-CD controls. Besides ourselves, only Gross and colleagues have previously evaluated the role of ASCA in NRCD [26]. The authors measured IgA class ASCA titers in 35 CD patients and 27 non-CD controls and further divided the $\mathrm{CD}$ group into GFD-responsive $(n=26)$ and nonresponsive $(n=9)$. They found the median ASCA titers in the NRCD patients to be quite similar $(17.3 \mathrm{U} / \mathrm{ml})$ to our figures $(14.5 \mathrm{U} / \mathrm{ml})$. In contrast, Gross and associates observed higher ASCA titers both in the untreated $(17.1 \mathrm{U} / \mathrm{ml}$ vs. $10.5 \mathrm{U} / \mathrm{ml}$, respectively) CD patients and GFD-responsive $(11.6 \mathrm{U} / \mathrm{ml}$ vs. $7.0 \mathrm{U} / \mathrm{ml})$ treated patients, and also in non-CD controls $(11.1 \mathrm{U} / \mathrm{ml}$ vs. $4.5 \mathrm{U} / \mathrm{ml})$. One plausible explanation for these discrepancies between studies might be use of different commercial kits for the ASCA analyses. Furthermore, the selection of patients and controls and the definition of NRCD were not identical. For instance, the patients in the study by Gross and group had been on a GFD for a markedly shorter time and appeared to be only slow responders, as they had ongoing mucosal damage during the study but recovered later during follow-up [26].

In neither the present study nor in that by Gross and colleagues did the ASCA titers differ significantly between NRCD and untreated CD, very likely because NRCD patients continued to evince histological damage in the small-bowel mucosa. In contrast, both we and they found ASCA titers to decrease in CD patients responding to GFD, and the same has also been reported in a few previous studies [15-17]. Moreover, we have shown a positive correlation between the severity of small-bowel mucosal damage and ASCA titers [17]. The gluten sensitivity of ASCA and its association with the histological damage is of particular interest regarding the follow-up of celiac disease, since tTG-ab have shown limited sensitivity in the assessment of mucosal recovery [27]. Hence, new sensitive and noninvasive biomarkers for monitoring GFD are being eagerly sought.

Interestingly, seropositivity to ASCA has been shown to be less frequent in children than in adults with $\mathrm{CD}$, very likely by reason of the shorter duration of gluten exposure and mucosal damage [16]. Further, even if increased, the ASCA titers in children also seem to decrease faster on GFD compared with adult patients [16]. On the other hand, RCD is practically nonexistent in children [28], indicating that the development of this severe condition requires longterm exposure to dietary gluten with ongoing small-bowel mucosal damage [4]. Gross and colleagues [26] investigated ten adults with type II RCD and found them to exhibit even higher serum ASCA titers than untreated CD patients, this finding supporting a possible relation between ASCA and disease severity. Interestingly, our data also showed a trend toward higher ASCA titers in NRCD than in CD at diagnosis. In fact in Crohn's disease, ASCA-positive patients have been reported to have a more aggressive clinical and endoscopic presentation with an increased risk of severe complications [11-13]. These findings indicate that positive ASCA signifies in general a more complicated disease course and poorer treatment response in both $\mathrm{CD}$ and IBD.

To our knowledge, this is the first study assessing serum antibodies to Pseudomonas fluorescens-associated sequence (I2) and Bacteroides caccae TonB-linked outer membrane protein $(\mathrm{OmpW})$ in NRCD patients. In contrast to ASCA, NRCD patients did not show significantly higher I2 or OmpW titers than those in the GFD-responsive group, while seropositivity to these two markers was more frequent in untreated CD than in the NRCD and other study groups. In Crohn's disease, seroreactivity toward I 2 is associated with severe fibrostenosing phenotype and a higher risk of small-bowel surgery [14]. Although we found I2 titers to be higher in NRCD than in healthy controls, there was no difference between the three $\mathrm{CD}$ groups, and the results do not support a major association between seroreactivity to I 2 and the development of NRCD. OmpW was elevated only in the CD groups other than NRCD when compared to controls, suggesting that it might take a longer time for OmpW titers to normalize during GFD.

It has been suggested that complicated interactions between various environmental and genetic factors and gut microbiota together modulate the risk for celiac disease [29]. The role of luminal microbiota in these circumstances is supported by the finding that its composition differs between active $\mathrm{CD}$ and healthy individuals [30]. Interestingly, we have recently shown treated CD patients with persistent symptoms to have intestinal dysbiosis and reduced microbial diversity in comparison with GFD-responsive patients [10], and the present observation of elevated microbial antibodies in NRCD could reflect a similar ongoing imbalance in the microbiota. Nevertheless, it still remains unclear whether there is a causal connection between intestinal dysbiosis and the persistency of CD activity, or whether the altered microbiota simply reflects an ongoing pathologic process triggered by other factors.

Another intriguing question is how the microbial antigens enter into the systemic circulation and drive the production of the corresponding serum microbial antibodies in different gastrointestinal conditions. In IBD, it has been suggested that they enter the body via a disrupted intestinal mucosal barrier [31]. Similarly, even treated CD patients may have increased intestinal permeability when compared to healthy controls [32], this possibly offering a route for microbial products to enter the circulation. On the other hand, in Crohn's disease ASCA titers do not necessarily correlate with intestinal permeability [33,34], and in the study by Gross and group no correlation was found between the degree of villous atrophy and mucosal permeability [26]. Moreover, we recently observed 
seroreactivity to microbial markers already at stages of $\mathrm{CD}$ prior to marked histological damage [18], suggesting that there could be some other mechanism than mucosal disintegration driving the elevated serum microbial antibody levels.

The main strengths of this research were the well-defined groups of NRCD and regular CD patients. In particular, the original $\mathrm{CD}$ diagnoses and medical information were confirmed as well as possible, and NRCD patients had undergone extensive exclusion of coexisting conditions possibly causing nonresponsivity. In addition, more than one microbial marker was included to obtain a broader view of the microbial seroreactivity in CD. In fact, neither I2 nor OmpW have previously been studied in patients with NRCD or RCD. As a limitation, the microbial markers were not measured in NRCD patients at diagnosis, and it thus remains unclear whether the titers would have differed from the responsive patients at that point. The duration of GFD was also shorter in the responsive than nonresponsive group which could have influenced the serological outcomes. Only two NRCD patients developed abnormal lymphocyte phenotype, and we thus had no true RCD group. It must also be emphasized that, although the NRCD patients underwent extensive diagnostic workup, some conditions such as food allergies were not systemically ruled out. In addition, the causal relationship between microbial markers and CD activity could not be evaluated in the present study design and thus remains a subject for further research.

To conclude, we found seropositivity and high titers of ASCA to be associated with NRCD. This indicates that ASCA is associated with more severe disease course and poorer response to GFD and might thus serve as an additional noninvasive marker of histological recovery in CD.

Acknowledgments We would like to acknowledge Jonathan Braun, M.D., Ph.D, Professor and Chair, Pathology and Laboratory Medicine, UCLA David Geffen School of Medicine, UCLA Health System for his support.

Funding This study was supported by the Academy of Finland, the Sigrid Juselius Foundation, the Päivikki and Sakari Sohlberg Foundation, the Competitive State Research Financing of the Expert Area of Tampere University Hospital, the Foundation for Pediatric Research, the Yrjö Jahnsson Foundation and State fund, Grant number Y1023G2017.

\section{Compliance with ethical standards}

Conflict of interest All authors declare that they have no conflict of interest.

\section{References}

1. Abdulkarim AS, Burgart LJ, See J, Murray JA. Etiology of nonresponsive celiac disease: results of a systematic approach. Am J Gastroenterol. 2002;97:2016-2021.
2. Leffler DA, Dennis M, Hyett B, Kelly E, Schuppan D, Kelly CP. Etiologies and predictors of diagnosis in nonresponsive celiac disease. Clin Gastroenterol Hepatol. 2007;5:445-450.

3. Stasi E, Marafini I, Caruso R, et al. Frequency and cause of persistent symptoms in celiac disease patients on a long-term gluten-free diet. J Clin Gastroenterol. 2016;50:239-243.

4. Ilus T, Kaukinen K, Virta LJ, et al. Refractory coeliac disease in a country with a high prevalence of clinically-diagnosed coeliac disease. Aliment Pharmacol Ther. 2014;39:418-425.

5. De Palma G, Nadal I, Medina M, et al. Intestinal dysbiosis and reduced immunoglobulin-coated bacteria associated with coeliac disease in children. BMC Microbiol. 2010;10:63.

6. Sartor RB, Wu GD. Roles for intestinal bacteria, viruses, and fungi in pathogenesis of inflammatory bowel diseases and therapeutic approaches. Gastroenterology. 2017;152:327.

7. Iltanen S, Tervo L, Halttunen T, et al. Elevated serum anti-I2 and anti-OmpW antibody levels in children with IBD. Inflamm Bowel Dis. 2006;12:389-394.

8. Ashorn S, Honkanen T, Kolho KL, et al. Fecal calprotectin levels and serological responses to microbial antigens among children and adolescents with inflammatory bowel disease. Inflamm Bowel Dis. 2009;15:199-205.

9. Landers CJ, Cohavy O, Misra R, et al. Selected loss of tolerance evidenced by Crohn's disease-associated immune responses to auto- and microbial antigens. Gastroenterology. 2002;123:689-699.

10. Wacklin P, Laurikka P, Lindfors K, et al. Altered duodenal microbiota composition in celiac disease patients suffering from persistent symptoms on a long-term gluten-free diet. Am J Gastroenterol. 2014;109:1933-1941.

11. Forcione DG, Rosen MJ, Kisiel JB, Sands BE. Anti-Saccharomyces cerevisiae antibody (ASCA) positivity is associated with increased risk for early surgery in Crohn's disease. Gut. 2004;53:1117-1122.

12. Papp M, Altorjay I, Dotan N, et al. New serological markers for inflammatory bowel disease are associated with earlier age at onset, complicated disease behavior, risk for surgery, and NOD2/CARD15 genotype in a Hungarian IBD cohort. Am J Gastroenterol. 2008;103:665-681.

13. Arnott ID, Landers CJ, Nimmo EJ, et al. Sero-reactivity to microbial components in Crohn's disease is associated with disease severity and progression, but not NOD2/CARD15 genotype. Am J Gastroenterol. 2004;99:2376-2384.

14. Mow WS, Vasiliauskas EA, Lin YC, et al. Association of antibody responses to microbial antigens and complications of small bowel Crohn's disease. Gastroenterology. 2004;126:414-424.

15. Granito A, Zauli D, Muratori P, et al. Anti-Saccharomyces cerevisiae and perinuclear anti-neutrophil cytoplasmic antibodies in coeliac disease before and after gluten-free diet. Aliment Pharmacol Ther. 2005;21:881-887.

16. Mallant-Hent RC, Mary B, von Blomberg E, et al. Disappearance of anti-Saccharomyces cerevisiae antibodies in coeliac disease during a gluten-free diet. Eur J Gastroenterol Hepatol. 2006;18:75-78.

17. Ashorn S, Välineva T, Kaukinen K, et al. Serological responses to microbial antigens in celiac disease patients during a glutenfree diet. J Clin Immunol. 2009;29:190-195.

18. Viitasalo L, Niemi L, Ashorn M, et al. Early microbial markers of celiac disease. J Clin Gastroenterol. 2014;48:620-624.

19. Rubio-Tapia A, Kelly DG, Lahr BD, Dogan A, Wu TT, Murray JA. Clinical staging and survival in refractory celiac disease: a single center experience. Gastroenterology. 2009;136:99-107.

20. Rubio-Tapia A, Murray JA. Classification and management of refractory celiac disease. Gut.. 2010;59:547-557. 
21. Taavela J, Koskinen O, Huhtala H, et al. Validation of morphometric analyses of small-intestinal biopsy readouts in celiac disease. PLoS One. 2013;8:e76163.

22. Järvinen TT, Kaukinen K, Laurila K, et al. Intraepithelial lymphocytes in celiac disease. Am J Gastroenterol. 2003;98:1332-1337.

23. Sutton CL, Kim J, Yamane A, et al. Identification of a novel bacterial sequence associated with Crohn's disease. Gastroenterology. 2000;119:23-31.

24. Wei B, Dalwadi H, Gordon LK, et al. Molecular cloning of a Bacteroides caccae TonB-linked outer membrane protein identified by an inflammatory bowel disease marker antibody. Infect Immun. 2001;69:6044-6054.

25. Kurppa K, Räsänen T, Collin P, et al. Endomysial antibodies predict celiac disease irrespective of the titers or clinical presentation. World J Gastroenterol. 2012;18:2511-2516.

26. Gross S, van Wanrooij RL, Tack GJ, et al. Antibody titers against food antigens decrease upon a gluten-free diet, but are not useful for the follow-up of (refractory) celiac disease. Eur J Gastroenterol Hepatol. 2013;25:516-518.

27. Kaukinen K, Sulkanen S, Mäki M, Collin P. IgA-class transglutaminase antibodies in evaluating the efficacy of gluten-free diet in coeliac disease. Eur J Gastroenterol Hepatol. 2002;14:311-315.

28. Mubarak A, Oudshoorn JH, Kneepkens CM, et al. A child with refractory coeliac disease. J Pediatr Gastroenterol Nutr. 2011;53:216-218.
29. Cenit MC, Olivares M, Codoner-Franch P, Sanz Y. Intestinal microbiota and celiac disease: cause, consequence or co-evolution? Nutrients. 2015;17(7):6900-6923.

30. Nistal E, Caminero A, Vivas $\mathrm{S}$, et al. Differences in faecal bacteria populations and faecal bacteria metabolism in healthy adults and celiac disease patients. Biochimie. 2012;94:1724-1729.

31. Zhou G, Song Y, Yang W, et al. ASCA, ANCA, ALCA and many more: are they useful in the diagnosis of inflammatory bowel disease? Dig Dis. 2016;34:90-97.

32. Vilela EG, Torres HO, Ferrari ML, Lima AS, Cunha AS. Gut permeability to lactulose and mannitol differs in treated Crohn's disease and celiac disease patients and healthy subjects. Braz J Med Biol Res. 2008;41:1105-1109.

33. Vermeire S, Peeters M, Vlietinck R, et al. Anti-Saccharomyces cerevisiae antibodies (ASCA), phenotypes of IBD, and intestinal permeability: a study in IBD families. Inflamm Bowel Dis. 2001;7:8-15.

34. Harrer M, Reinisch W, Dejaco C, et al. Do high serum levels of anti-Saccharomyces cerevisiae antibodies result from a leakiness of the gut barrier in Crohn's disease? Eur J Gastroenterol Hepatol. 2003;15:1281-1285.

\section{Affiliations}

\section{Liisa Viitasalo $^{1,2} \cdot$ Kalle Kurppa $^{1}$ (D) Merja Ashorn ${ }^{1} \cdot$ Päivi Saavalainen ${ }^{3} \cdot$ Heini Huhtala $^{4} \cdot$ Sara Ashorn $^{1}$. Markku Mäki ${ }^{1} \cdot$ Tuire Ilus $^{5} \cdot$ Katri Kaukinen $^{6,7} \cdot$ Sari Iltanen $^{1,8}$}

Liisa Viitasalo

viitasalo.liisa.m@student.uta.fi

Päivi Saavalainen

paivi.saavalainen@helsinki.fi

Heini Huhtala

heini.huhtala@uta.fi

Sara Ashorn

ashorn.sara.m@student.uta.fi

Markku Mäki

markku.maki@uta.fi

Tuire Ilus

tuire.ilus@pshp.fi

Katri Kaukinen

katri.kaukinen@uta.fi

Sari Iltanen

sari.iltanen@uta.fi

1 Tampere Center for Child Health Research, University of Tampere and Tampere University Hospital, Arvo Ylpön katu 34, 33014 Tampere, Finland
2 Department of Clinical Genetics and Laboratory of Genetics, HUSLAB, University of Helsinki and Helsinki University Hospital, 00029 Helsinki, Finland

3 Research Programs Unit, Immunobiology, and Department of Medical and Clinical Genetics, University of Helsinki, P.O. Box 21, Haartmaninkatu 3, 00014 Helsinki, Finland

4 Faculty of Social Sciences, University of Tampere, P.O. Box 100, 33014 Tampere, Finland

5 Department of Gastroenterology and Alimentary Tract Surgery, Tampere University Hospital, P.O. Box 2000, Tampere 33521, Finland

6 Celiac Disease Research Center, Faculty of Medicine and Life Sciences, University of Tampere, P.O. Box 100, 33014 Tampere, Finland

7 Department of Internal Medicine, Tampere University Hospital, P.O. Box 2000, 33521, Tampere, Finland

8 Lapland Central Hospital, P.O. Box 8041, 96101 Rovaniemi, Finland 